\title{
The PHC-cog: A brief cognitive function screening test for the elderly
}

\author{
Moon Ho Park, Dae Hie Lee, Hong Jae Lee*, Mi Sook Song** \\ Department of Neurology, Korea University Medical College, Seoul, "Bundang Public Health Center, Sungnam-si, Gyeoggi-do, \\ ${ }^{* \star D}$ Department of Nursing Science, Ajou University Medical College, Suwon, Gyeonggi-do, Korea
}

\begin{abstract}
Background: Cognitive dysfunctions such as dementia have emerged as a leading public health problem among the elderly. Its early detection is important for the management of patients, and for the educational support of other family members. Although cognitive dysfunction screening tests are available, they have not been widely utilized in community or primary care settings. Aim: To validate the PHC-cog (Public Health Center Cognitive Dysfunction Test) and to assess it as a simple and brief method for massive screening of cognitive dysfunctions in the primary care setting. Materials and Methods: The study comprised $137 \mathrm{com}$ munity-dwelling patients with dementia and 134 age, sex, and education-matched controls. The PHC-cog was made combining patients' and informants' questionnaires to improve performance. The PHC-cog patient's section briefly assessed the patient's cognition. Results: The PHC-cog Patient's Section had a sensitivity and specificity of 0.75 and 0.92 , respectively. The PHC-cog Informants' Section had a sensitivity and specificity of 0.79 and 0.83 , respectively. The total method of administering the PHC-cog had a sensitivity and specificity of 0.96 and 0.82 , and the two-stage method had a sensitivity and specificity of 0.92 and 0.76 , respectively. Conclusions: The PHC-cog test is a simple, accurate and reliable, performance-based tool in the screening for cognitive dysfunction. The PHC-cog test is quick, and easy-to-use, and will hopefully become widely used in the cognitive screening of the aging population in the primary public medical institutions of Korea.
\end{abstract}

Key Words: Cognitive function, Brief Screening test, PHC$\operatorname{cog}$

\section{Introduction}

The need for an efficient and effective cognitive screening method has become more important due to its rightful appli- cation as a discreet pre-medical system, which will allow the elderly to easily receive a cognitive function test. The use of cognitive screening tools to detect dementia and to determine the status of the cognitive function is now one of the fundamental health care procedures for the elderly.

Currently, the Mini-Mental State Examination (MMSE), ${ }^{[1]}$ the Mini- $\operatorname{Cog}^{[2]}$ and the General Practitioner Assessment of Cognition (GPCOG) ${ }^{[3]}$ are being used widely for cognitive screening. Since these tests are relatively complicated and timeconsuming, it is difficult to use them for massive screening or epidemiological studies of community-dwelling old people. The screening or epidemiological study of cognitive dysfunction should be culturally fair, psychometrically sound, and valid for some specific considerations in the study population, such as educational variances. ${ }^{[4,5]}$ Public medical institutions in developing countries are likewise not effectively organized, and have insufficient manpower and funds to be able to administer such complicated, time-consuming, and costly tests to the elderly as part of their primary health care services. A brief screening test that can be used with accuracy and simplicity is, therefore, an essential tool in the examination of old people who visit primary care agencies, or as a public health service for those living in communities. ${ }^{[2,3]}$ For this purpose, a brief cognitive function screening test was developed, which can be used as a first-line medical tool in public health centers (PHCs), which are public medical institutions in Korea.

\section{Materials and Methods}

Every year, PHCs in Korea administer free influenza vaccinations across the country to people aged 65 and over. Influenza vaccinations at PHCs, however, do not have the latitude to test the cognitive function of older people within a short time, and there is insufficient medical manpower at the PHCs to do so. A new instrument, the Public Health Center Cognitive Dysfunction Screening Test (PHC$(\mathrm{cog})$ was therefore tested for the detection of dementia in public medical institutions. 


\section{Development of the PHC-cog}

To quickly, conveniently and simply yet effectively detect cognitive dysfunction in the elderly, the PHC-cog was developed by supplementing informant questionnaires with patient questionnaires, the combination of which can increase predictive power. ${ }^{[6]}$ Items were derived from four sources: the Mini Mental State Examination, ${ }^{[1]}$ the Barthel Index, ${ }^{[7]}$ the Instrumental Activities of Daily Living $(\mathrm{IADL}),{ }^{[8]}$ and the Korean Dementia Screening Questionnaire $(\mathrm{KDSQ}) \cdot{ }^{[9]}$

The PHC-cog Patient's Section was prepared in such a way that the questionnaires could be completed within a short time, so that as many patients as possible could be examined. This addresses a specific need of developing countries. Geriatric neurologists and nurses, who generally manage dementia patients, put together items on the memory and other cognitive functions. The PHC-cog Patient's Section consists of ten cognitive test items. Scoring is based on the total number of incorrect responses, the maximum score is 20, and lower scores indicate better functions. A refined PHC-cog Patient's Section consisting of five cognitive items was subsequently developed (see results).

In the PHC-cog Informants' Section, the cognition-related sections of the KDSQ ${ }^{[9]}$ which were widely adopted in Korea, were used. The cognition-related questions of the KDSQ were intended to check out the memory functions, the language and other cognitive functions, and dysfunction of the complex task performance faculty. The PHC-cog Informants' Section is calculated using the total number of scale points, the maximum score was 30 , and lower scores indicated better functions.

\section{Subjects}

All subjects who visited the Bundang-gu PHC from April 2003 to March 2004 were recruited. Among the dementia patients who visited the Bundang-gu PHC geriatric clinic, 137 completed the study. All the patients fitted in with "the derivation of criterion standards, Diagnostic and Statistical Manual of Mental Disorders (Fourth Edition) (DSM-IV), diagnoses of dementia." ${ }^{[10]}$ The average age of the patients was $74.23 \pm 5.57$ and $94(68.6 \%)$ of them were females. The level of education of the patients was $8.37 \pm 5.91$ years. Among those who visited the flu clinic, 134 controls with normal cognitive functions were selected by age, sex, and education-matched methods to evaluate the validity and reliability of the PHC-cog. All controls did not fit in with DSM-IV diagnosis of dementia. ${ }^{[10]}$ The average age of controls was $74.07 \pm 5.43$ and $92(68.7 \%)$ of them were females. The level of education was $8.36 \pm 5.84$ years. Controls did not differ from patients as regards age $(t=0.238, d f=238, P=0.812)$, sex $\left(\chi^{2}=0.994, d f=1, P=0.549\right)$, or education $(t=0.020, d f=238$, $P=0.984)$. When the clinical dementia rating $(\mathrm{CDR})^{[11,12]}$ was administered to all participants, patients were rated more than CDR 1 and controls were rated CDR 0.

The study was approved by the appropriate research ethics committee. Informed consent was obtained from all participants or their relatives.

\section{Evaluation of reliability and examination of validity of the PHC-cog}

To evaluate the validity of the PHC-cog through its interrelationship with its variables that are related to other cognitive tests, MMSE $^{[1,13]}$ was administered to the patients and controls. To evaluate the day-to-day activities, $\mathrm{LADL}$ test ${ }^{[8,14]}$ was administered to them.
This study used the Korean version of IADL. ${ }^{[14]}$ Possible scores on the Korean version of IADL range from 0 to 3 with higher values indicative of greater impairment.

The PHC-cog Informants' Section was accomplished by informants of the patients, who were limited to those who were well aware of the patient's status through cohabiting or regular visits with them of at least three times a week. The informants were instructed on how to complete the PHC-cog Informants' Section and were asked for firsthand entries. All tools were evaluated and interpreted blindly to subject data.

\section{Statistical analysis}

For demographic and clinical characteristics, differences on continuous data were assessed using independent samples $t$-tests. Chisquare tests of significance were used to test for significant associations between cross-tabulated data, and exact tests were used where appropriate.

Pearson's correlations were used to assess associations between continuous variables. Statistical tests were 2 -tailed and results were regarded as significant at or below the $5 \%$ probability level.

Receiver operator characteristic (ROC) analyses were used to assess the PHC-cog as a screening tool. The areas under the curves (AUCs) were compared, which represent the probability that the patients and controls were correctly ranked by the test according to their diagnostic status.

\section{Results}

The MMSE scores of the patients were $18.83 \pm 6.55$ and of the controls, 26.85 \pm 2.80 , showing a significant lower score for the patients $(t=-13.044, d f=269, P=0.001)$. The IADL scores of the patients were $0.57 \pm 0.62$ and of the controls, $0.12 \pm 0.18$, showing a significant difference $(t=8.103$, $d f=269, P=0.010)$.

The PHC-cog Patient's Section subscales were refined by eliminating items that were completed incorrectly by fewer than $5 \%$ of patients, or those that did not assist in the discrimination of dementia-diagnostic prediction as determined by logistic regression analyses. The PHC-cog Informant's Section was used like the original version of KDSQ cognition section. Thus, the refined PHC-cog, consisting of the 5-item Patient's Section and the 15-item Informants' Section, was used for subsequent analyses, unless otherwise specified.

In the PHC-cog Patient's Section, the interrater intraclass correlation coefficient (ICC) was $0.79(P=0.010)$, the testretest ICC was $0.85(P=0.001)$, and the internal consistency (Cronbach's á) was 0.63. In the PHC-cog Informants' Section, the test-retest ICC was $0.83(P=0.020)$, and the internal consistency (Cronbach's á) was 0.72 , which were similar to the existing results. ${ }^{[9]}$ Thus, both the Patient's and the Informants' Sections of the PHC-cog showed satisfactory reliability and validity.

Sensitivities and specificities were examined for the two subsection scores, separately and together (total method and twostage method) (Table 1). The total method was simply the calculated total score plus both the Patient's and Informants' 


\begin{tabular}{|c|c|c|c|c|c|}
\hline Variable & $\begin{array}{c}\text { PHC-cog } \\
\text { patients' } \\
\text { section }\end{array}$ & $\begin{array}{c}\text { PHC-cog } \\
\text { informants' } \\
\text { section }\end{array}$ & $\begin{array}{c}\text { PHC-cog } \\
\text { total } \\
\text { method }\end{array}$ & $\begin{array}{c}\text { PHC-cog } \\
\text { two-Stage } \\
\text { method }\end{array}$ & MMSE \\
\hline Maximum score & 10 & 30 & 40 & & 30 \\
\hline \multirow[t]{4}{*}{ Cut-off point (Sensitivity/Specificity) } & $4(0.89 / 0.65)$ & $4(0.94 / 0.66)$ & $6(0.98 / 0.66)$ & ${ }^{*}(0.92 / 0.76)$ & $19(0.47 / 0.98)$ \\
\hline & *6 (0.75/0.92) & $5(0.89 / 0.74)$ & *8 (0.96/0.82) & & $21(0.57 / 0.93)$ \\
\hline & $8(0.05 / 0.99)$ & *6 $(0.79 / 0.83)$ & $10(0.84 / 0.90)$ & & $23(0.74 / 0.87)$ \\
\hline & $10(0.03 / 1.00)$ & $7(0.64 / 0.91)$ & $12(0.68 / 0.96)$ & & $25(0.88 / 0.76)$ \\
\hline AUCs & 0.91 & 0.88 & 0.95 & 0.90 & 0.89 \\
\hline AUCs $95 \%$ confidence interval & $0.87-0.95$ & $0.84-0.92$ & $0.93-0.98$ & $0.86-0.94$ & $0.86-0.93$ \\
\hline Standard error of AUCs & 0.018 & 0.021 & 0.012 & 0.019 & 0.018 \\
\hline
\end{tabular}

*Optimal cut-off point on the ROC curve farthest from the diagonal line.

Section scores. The two-stage method was a simple addition method, which diagnosed a subject as having dementia when the PHC-cog Patient's or Informants' Section indicated them as cognitive-impaired. In the PHC-cog Patient's Section, sensitivity was 0.75 and specificity was 0.92 when dementia was diagnosed for the higher section of 6 points on the ROC curve farthest from the diagonal line. In the PHC-cog informants' section, sensitivity was 0.79 and specificity was 0.83 when dementia was diagnosed for scores of 6 or higher. Both the PHC-cog Patient's Section and Informants' Section showed comparatively high sensitivity and specificity results. In addition, the PHC-cog total method showed a slightly poorer specificity, but it showed an improved sensitivity when used to determine cognitive dysfunction. The PHC-cog two-stage method showed almost same sensitivity and specificity.

To find out the interrelationship between the PHC-cog and the existing screening test methods, their Pearson's correlations were examined. The PHC-cog Patient's Section and MMSE and LADL scores were correlated $(\mathrm{r}=-0.674, P=0.001$ and $r=0.508, P=0.001$, respectively), which indicated a significant interrelationship. The PHC-cog Informants' Section and MMSE and IADL scores were correlated $(\mathrm{r}=0.568$, $P=0.025$ and $\mathrm{r}=-0.566, P=0.043$, respectively), which were also found to be statistically significant. To determine agreement within the PHC-cog, the Patient's Section and the Informants' Section were examined. The PHC-cog Patient's Section was significantly correlated with the Informants' Section $(\mathrm{r}=0.580, P=0.010)$, the total method $(\mathrm{r}=0.724$, $P=0.012)$, and the two-stage method $(r=0.509, P=0.023)$. The PHC-cog Informants' Section was significantly correlated with the total method $(\mathrm{r}=0.735, P=0.043)$ and the two-stage method ( $\mathrm{r}=0.733, P=0.001)$, and the PHC-cog total method was significantly correlated with the two-stage method $(\mathrm{r}=0.707, P=0.010)$.

To determine the influence of age, gender, and education on the PHC-cog, a stepwise multiple linear regression analysis was used in the controls. For the PHC-cog Patient's Section, age and gender were not related, but only the level of education $\left(\mathrm{R}^{2}=0.07, B=0.068, t=-28.76, P=0.045\right)$ was revealed to have a relation. The PHC-cog Informants' Section, however, was not related to age, gender, and educational level
$\left(\mathrm{R}^{2}=0.03, P>0.05\right)$. For scoring methods, the total method $\left(\mathrm{R}^{2}=0.03, P>0.05\right)$ and the two-stage method $\left(\mathrm{R}^{2}=0.02\right.$, $P>0.05$ ) were not influenced to age, gender, and level of education.

The ROC curves were obtained (Table 1). The AUCs scored 91\% in the PHC-cog Patient's Section, 88\% in the PHC-cog Informants' Section, $95 \%$ in the total method, $90 \%$ in the two-stage method, and $89 \%$ in MMSE. Overall, when compared with MMSE, the PHC-cog total method and two-stage method showed similar results. The ROC analysis showed that there was no significant difference between the AUCs of the total method and the two-stage method $\left(\chi^{2}=188.26, d f=1\right.$, $P>0.05)$.

Post hoc, the ability of the MMSE, using the recommended cut-off point of $23 / 24,{ }^{[1]}$ was compared to that of the total method and the two-stage method in detecting cases of dementia. The sensitivity, specificity, and AUC were similar to those of the PHC-cog since the AUC did not significantly differ from the total method $\left(\chi^{2}=56.71, d f=1, P>0.05\right)$ and from the two-stage method $\left(\chi^{2}=73.45, d f=1, P>0.05\right)$. These analyses lend additional weight to the validity of the PHC$\operatorname{cog}$.

The administration time for the PHC-cog Patient's Section, given as a single test, was $1.01 \pm 0.73$ minutes. And that for the PHC-cog Informants' Section was $4.50 \pm 2.65$ minutes. However, the MMSE required $7.45 \pm 5.33$ minutes.

\section{Discussion}

This study attempted to develop a brief screening test for the detection of cognitive dysfunction, which can be quickly administered by laymen in places where medical manpower is limited, and which does not require professional skills to interpret its results.

One of the strengths of the PHC-cog lies in its inclusion of informant data. It is important to have informant data in cognitive dysfunction screening. The diagnosis of dementia is also based on the information provided by informants, such as family. Information from patients' descendants with comparatively high educational backgrounds is especially significant in testing the elderly in developing countries, most of whom have 
had low levels of education. These descendants can fill up the Informants' Section in advance, and submit it to the physician on the day of the diagnosis through the subjects, without having to visit a medical institution.

In this study, the PHC-cog has been validated in a primary care setting. The scores of the patients and the controls displayed noticeable differences in the PHC-cog Patients' and Informants' Sections. The Pearson's correlation of the PHC$\operatorname{cog}$ was compared with that of MMSE and IADL to evaluate for efficiency. The measurement of the cognitive functions of the elderly was very often influenced by psychological and environmental factors. The results of the cognitive function tests on the subjects, who were old people suffering from cognitive dysfunction, largely depended on their psychological status, the attitude of the examiner, and the surrounding environment. ${ }^{[15]}$ For these reasons, evaluating the inter-rater ICC and the test-retest ICC are essential parts in the verification of the reliability of these tests, since they provide an evaluation of the level of influence that produces an impact on the test results. Both the inter-rater ICC and the test-retest ICC displayed comparatively high levels of reliability in this study.

When evaluating the diagnostic validity of a new screening test, it is very critical to know which score should be chosen as the base to sort out cognitive dysfunction. This can be determined by calculating the sensitivity and specificity of each cut-off point. The result of using the ROC curve for the optimum cut-off point, which satisfies both sensitivity and specificity for dementia diagnosis based on the validity of the PHC-cog for subjects, in comparison to the reference standard for DSM-IV, was 0.82 for sensitivity and 0.96 for specificity in the PHC-cog total method. The ROC curve analysis was used to compare the diagnostic efficiency between the diagnoses derived from the AUCs calculation, other than the estimate of the cut-off point, and the test method. While AUCs of 0.85 or more are considered an excellent test method in general, this study revealed AUCs of 0.95 for the PHC-cog total method.

The advantages of the PHC-cog over the current brief screening tools are that they combine patient and informant data, and are quick and easy for public health institutions to use. The PHC-cog effectively screens a number of patients within a short time. The testing time of the Patient's Section was less than one-seventh of that required for the MMSE, and that of Informants' Section was also less than that of the MMSE. Its cost in materials (paper and pencil) is negligible.

This study suggested two scoring methods: the total method and the two-stage method. In the total method, the sensitivity, specificity, and AUCs were most excellent. Because there are many living-alone elderly people who cannot be assessed by an informant, their cognitive functions can be evaluated by the two-stage method or by the Patient's section.

There are many distinctive features of the PHC-cog. First, it is easy to use, as it has fewer and simpler questions. Sec- ond, laymen can quickly administer the test and it does not require professional skills to interpret its results. In most developing countries, public medical institutes which are the primary medical services for community-dwelling people, are not only insufficient but also have a limited number of clinicians. For the primary assessment or screening of cognitive dysfunctions, such as dementia, in a primary care setting, the use of relatively brief screening tests that require only minimal training for administration, that are not time-consuming and expensive, is required. These brief screening tests can be used at public medical institutes in developing countries. Third, the PHC-cog is a comparatively superior method in terms of its reliability and validity, and is a handy method that saves time and energy.

In conclusion, this brief screening tool, the PHC-cog, although not the sole screening tool for dementia, is considered comparatively accurate in determining the decline of the cognitive function, resulting in dementia from the normal cognitive function. Furthermore, any trained person in primary public medical institutions catering to community residents can administer it to the elderly quickly, easily, and reliably.

\section{References}

1. Folstein MF, Folstein SE, McHugh PR. "Mini-mental state". A practical method for grading the cognitive state of patients for the clinician. J Psychiatr Res $1975 ; 12: 189-98$.

2. Borson S, Scanlan J, Brush M, Vitaliano P, Dokmak A. The mini-cog: A cognitive 'vital signs' measure for dementia screening in multi-lingual elderly. Int .J Geriatr Psychiatry 2000;15:1021-7.

3. Brodaty H, Pond D, Kemp NM, Luscombe G, Harding L, Berman K, et al The GPCOG: A new screening test for dementia designed for general practice. J Am Geriatr Soc 2002;50:530-4.

4. Chandra V, Ganguli M, Ratcliff G, Pandav R, Sharma S, Belle S, et al. Practical issues in cognitive screening of elderly illiterate populations in developing countries. The Indo-US Cross-National Dementia Epidemiology Study. Aging (Milano) 1998;10:349-57.

5. Chandra V, Ganguli M, Rateliff G, Pandav R, Sharma S, Gilby J, et al. Studies of the epidemiology of dementia: comparisons between developed and developing countries. Aging (Milano) 1994;6:307-21.

6. Mackinnon A, Mulligan R. Combining cognitive testing and informant report to increase accuracy in screening for dementia. Am J Psychiatry 1998;155:1529-35.

7. Mahoney FI, Barthel DW. Functional Evaluation: The Barthel Index. Md State Med J 1965;14:61-5.

8. Lawton MP, Brody EM. Assessment of older people: Self-maintaining and instrumental activities of daily living. Gerontologist 1969;9:179-86.

9. Yang DW, Cho B, Chey JY, Kim SY, Kim BS. The development and validation of Korean Dementia Screening Questionnaire (KDSQ). J Korean Neurol Assoc $2002 ; 20: 135-41$.

10. American Psychiatric Association. Diagnostic and Statistical Manual of Mental Disorders (Fourth Edition) (DSM-IV). $4^{\text {th }}$ Ed. Washinton, DC: APA; 1994.

11. Morris JC. The Clinical Dementia Rating (CDR): Current version and scoring rules. Neurology 1993;43:2412-4.

12. Choi SH, Na DL, Lee BH, Hahm DS, Jeong JH, Yoon SJ, et al. Estimating the validity of expanded clinical dementia rating (CDR) scale. J Korean Neurol Assoc 2001;19:585-91.

13. Kang Y, Na DL, Hahn S. A validity study on the Korean Mini-Mental State Examination (K-MMSE) in dementia patients. J Korean Neurol Assoc 1997;15:300-7.

14. Kang SJ, Choi SH, Lee BH, Kwon JC, Na DL, Han SH, et al. The reliability and validity of the Korean instrumental activities of daily living (K-LADL). J Korean Neurol Assoc 2002;20:8-14.

15. Morris JC. Handbook of dementing illness. $1^{\text {st }}$ Ed. New York: Marcel Dekker; 1994.

Accepted on 06.11.2004. 


\section{A. Patients' Section}

Question

1. What kind of vaccination do you want?

2 . What date of the month is it today?

3. What day of the week is it today?

4. When is your birthday?

5. Interlocking pentagon

\section{B. Informants' Section}

Question

1. Is the subject unaware about what date of the month and what day of the week it is today?

2. Is the subject able to retrieve his/her own items?

3. Does the subject repeat a question?

4. Does the subject forget appointments?

5. Does the subject come back without retrieving the item he/she went for?

6. Does the subject hesitate whenever he/she is trying to recall a person's name or the things he/she wants?

7. Does the subject ask repeatedly about the same part of a dialogue due to his/her inability to understand it?

8. Has the subject once lost his/her direction and wandered about?

9. Has the subject's ability to calculate failed compared to the past?

10. Has the subject's personality changed compared to the past?

11. Is the subject clumsy with tools that he/she skillfully used in the past?

12. Does the subject keep rooms in his/her house or his/her house itself untidy as compared with in the past?

13. Does the subject improperly select clothes for an event?

14. Does the subject find it difficult to reach his/her destination by using public transportation? (Exclude cases arising from physical disorder)

15. Is the subject unable to change his/her clothes when they are already dirty?
Answer score (0/2)

(Flu / Don't know)

( Correct/Wrong )

( Correct/Wrong )

( Correct/Wrong )

( Correct/Wrong )

(two pentagon have to be precisely interlocked)

Answer

(No / Sometimes, Yes / Very often yes)

Score
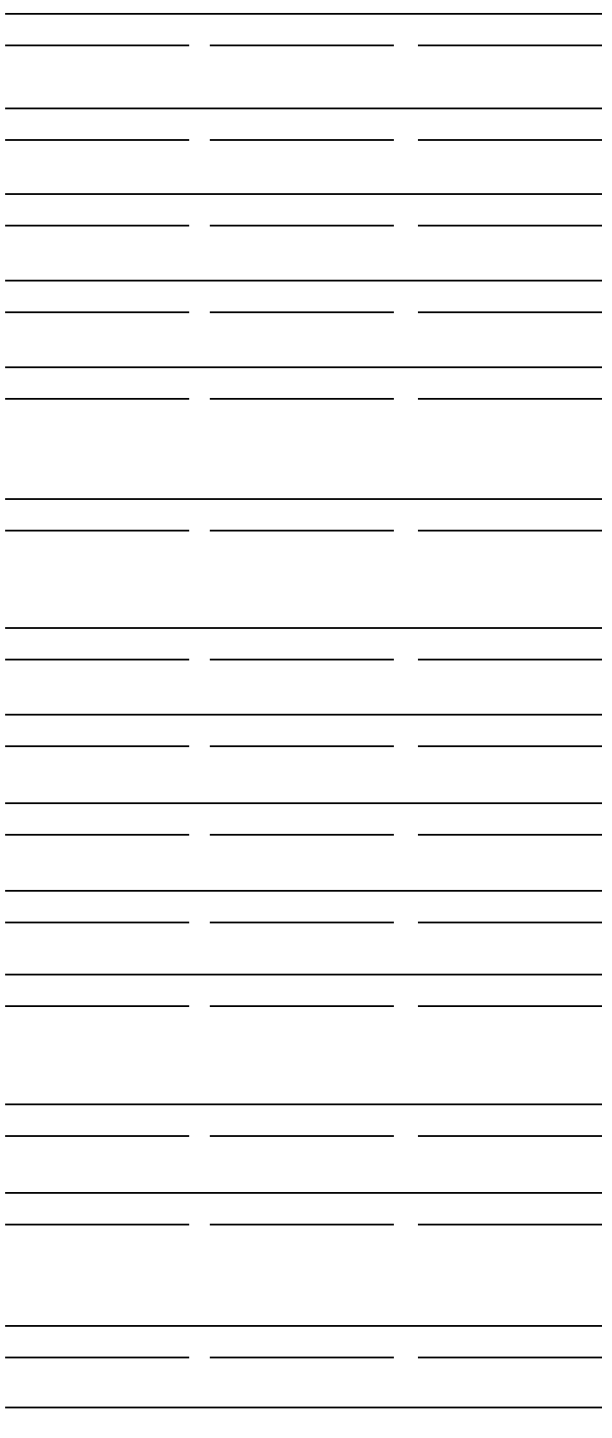


\section{Invited Comments}

There are not too many options for the assessment of cognitive functions in the elderly or in whom you suspect dementias. The options are further limited if you have busy clinics, too many patients and a lack of time and personnel. The PHCCog attempts to be user-friendly and of assistance to clinicians to do rapid assessments to screen cases of cognitive dysfunction. They could do it themselves or train any lay person or another staff member to do it. This study has attempted to develop this brief screening test for the detection of cognitive dysfunction, which can be quickly administered by laymen in places with limited medical manpower, and which does not require professional skills to interpret its results.

The PHC-Cog relies heavily on time disorientation items as a screening instrument. These items were found to have the best discriminating ability for diagnostic prediction of dementia. It is known that time sense orientation gets affected quite early, and hence the use of such items may pick up early cases. The psychometric properties have been found to be sound. The 'Informant's section' is a little more detailed, but still contains statements which are usually reported by the relatives of the dementia patients. The PHC-Cog does not have items related to recent memory disturbances.

The screening instrument can only be used if the elderly subjects visit the health center for a flu vaccination! The authors need to suggest some flexible item which can substitute this, otherwise it will restrict its usage. Some illiterate Indian patients may find it difficult to understand the interlocking of the pentagons. The 'Informant's section' also has some items which may be potentially problematic - like the one about keeping appointments, change of personality, or selection of clothes.

This One-minute screening instrument is promising for detection at the primary care level or for community surveys. It has been tried out in Korea. Other developing countries and busy centers need to try it out to actually determine the scale's usefulness. The scale would need some adaptation for Indian settings and for different health centers. For example, if the person has come for a check-up of his blood pressure or blood sugar, or some other aspect, that could become the first question, replacing flu vaccination. It also has potential for usage to detect cognitive dysfunction due to causes other than dementia. The screening instrument does not show any indication of being used as a prognostic tool though, and the authors have made no such claims.

Santosh K. Chaturvedi

National Institute of Mental Health and Neurosciences, Bangalore, India.E-mail: skchatur@rediffmail.com 